ARTICLE

\title{
Mutant-selective degradation by BRAF-targeting PROTACs
}

\author{
Shanique Alabi ${ }^{1}$, Saul Jaime-Figueroa ${ }^{2}$, Zhan $\mathrm{Yao}^{3}$, Yijun Gao ${ }^{3}$, John Hines ${ }^{2}$, Kusal T. G. Samarasinghe ${ }^{2}$, \\ Lea Vogt ${ }^{2}$, Neal Rosen (D) $^{3}$ \& Craig M. Crews (iD) ${ }^{1,2,4 凶}$
}

Over 300 BRAF missense mutations have been identified in patients, yet currently approved drugs target $\mathrm{V} 600$ mutants alone. Moreover, acquired resistance inevitably emerges, primarily due to RAF lesions that prevent inhibition of BRAF V600 with current treatments. Therefore, there is a need for new therapies that target other mechanisms of activated BRAF. In this study, we use the Proteolysis Targeting Chimera (PROTAC) technology, which promotes ubiquitination and degradation of neo-substrates, to address the limitations of BRAF inhibitor-based therapies. Using vemurafenib-based PROTACs, we achieve low nanomolar degradation of all classes of BRAF mutants, but spare degradation of WT RAF family members. Our lead PROTAC outperforms vemurafenib in inhibiting cancer cell growth and shows in vivo efficacy in a Class 2 BRAF xenograft model. Mechanistic studies reveal that BRAFWT is spared due to weak ternary complex formation in cells owing to its quiescent inactivated conformation, and activation of BRAFWT sensitizes it to degradation. This study highlights the degree of selectivity achievable with degradation-based approaches by targeting mutant BRAF-driven cancers while sparing BRAFWT, providing an anti-tumor drug modality that expands the therapeutic window.

\footnotetext{
${ }^{1}$ Department of Pharmacology, New Haven, CT, USA. ${ }^{2}$ Molecular, Cellular, and Developmental Biology, Yale University, New Haven, CT, USA. ${ }^{3}$ Program in Molecular Pharmacology, Memorial Sloan Kettering Cancer Center, New York, NY, USA. ${ }^{4}$ Department of Chemistry, Yale University, New Haven, CT, USA

凶email: craig.crews@yale.edu
} 
$\mathrm{T}$ he Ras-RAF-MEK-ERK pathway is important for many aspects of cellular homeostasis ${ }^{1}$. The pathway is initiated upon extracellular growth factor binding to receptor tyrosine kinases (RTKs), thereby activating the kinase cascade ${ }^{2}$. Upon activation of upstream effectors, GTP-bound RAS recruits RAF (ARAF, BRAF, or CRAF) to the cell membrane, promoting its dimerization and activation ${ }^{2}$. Thus, the scaffolding and enzymatic role of BRAF are both essential for its function ${ }^{3-5}$. Activated RAF phosphorylates and activates MEK, which in turn phosphorylates and activates ERK leading to cell proliferation, differentiation, and survival ${ }^{2}$. BRAF is mutated in $8 \%$ of observed cancers including melanoma $(60 \%)^{6}$, colorectal cancer $(10 \%)^{7}$, non-small cell lung cancer (NSCLC) $(10 \%)^{8}$, and hairy cell leukemia $(100 \%)^{9}$. These mutations (often missense mutations found in the kinase domain) distinctly affect the biochemical characteristics of the kinase ${ }^{10-12}$. Class 1 BRAF mutants such as V600E and V600K are hyperactivating and can signal as monomers in the absence of activated RAS $^{13}$. Class 2 BRAF mutants such as K601E and G469A signal as constitutive, RAS- independent dimers ${ }^{14}$. Lastly, Class 3 BRAF mutants such as G466V and D594N harbor low to no kinase activity and function by binding tightly to RAS thus recruiting CRAF into hyperactivated heterodimers ${ }^{15-17}$. FDA-approved inhibitors such as vemurafenib have been successful in increasing progression-free survival of patients harboring hyperactive BRAF $\mathrm{V} 600 \mathrm{E}$ mutations ${ }^{18}$. However, as with many kinase inhibitors, resistance occurs that renders patients insensitive to continued treatment ${ }^{19}$. Significant efforts have focused on creating drugs that target Class 2 BRAF mutations by inhibiting dimer formation, but adequate drugs have not yet been approved ${ }^{10}$. Therefore, there is a need for new and innovative therapies to address BRAF-driven cancers.

Proteolysis Targeting Chimeras (PROTACs) are heterobifunctional small molecules composed of a warhead that binds a protein of interest (POI), a flexible linker, and a ligand that binds an E3 ligase ${ }^{20,21}$. These molecules recruit an E3 ligase (e.g., VHL) to a POI to form a ternary complex. Upon complex formation, ubiquitin molecules are transferred to accessible lysines on the POI, marking it for proteasomal degradation. Importantly, by eliminating the entire protein scaffold, PROTACs are able to target both the enzymatic and non-enzymatic roles of diseasecausing proteins. In recent years, our lab and others have made considerable progress in using PROTAC technology to induce degradation of proteins involved in disease, such as AR, ER, BRD4, RIPK2, BCR-Abl, EGFR, MET, p38 MAPK, BTK, and $E R R a^{22-32}$. While traditional inhibitors require sustained target engagement for therapeutic effect, PROTACs simply require transient interaction, offering the ability to degrade proteins with limited target engagement ${ }^{22}$. Furthermore, the modular design of PROTACs allows for additional selectivity to be tuned into the small molecule, making it ideal for addressing difficult targets such as BRAF.

In this work, we use targeted protein degradation as a strategy to address mutant BRAF-driven cancer. We hypothesize that incorporating a BRAF inhibitor into PROTACs would allow for degradation of all BRAF isoforms. Despite the parent inhibitor, vemurafenib, being specific for Class 1 mutants only, we find that our PROTACs induce degradation of all three classes of BRAF mutants. Interestingly, $\mathrm{BRAF} \mathrm{WT}^{\mathrm{WT}}$ is degraded to a far lesser extent, and mechanistic studies show that $\mathrm{BRAF}^{\mathrm{WT}}$ is spared due to a weaker ternary complex with the PROTAC and E3 ligase in cells. Furthermore, we are able to tune BRAFWT degradation by increasing WT BRAF kinase activity. Overall, this study shows selective targeting of mutant BRAF with PROTAC technology and explores the mechanism that underlies the observed selectivity.

\section{Results}

SJF-0628 induces efficient and potent degradation of mutant BRAF but spares BRAF ${ }^{W T}$. Although the utility of vemurafenib is limited to the treatment of tumors driven by BRAFV600 mutations, biochemical and binding studies show that these inhibitors also interact with BRAFWT, Class 2, and Class 3 BRAF mutants ${ }^{11,15,18,33}$. We therefore hypothesized that all BRAF isoforms would be susceptible to degradation by a vemurafenibbased PROTAC. Crystal structures of vemurafenib bound to $\mathrm{BRAF}^{\mathrm{V} 600 \mathrm{E}}$ reveal a solvent-exposed chloride at the para-position on the phenyl ring, which we posited would be ideal for linker addition (PDB: 3OG7) ${ }^{34}$ (Supplementary Fig. 1a,b). Pursuant to this, we iteratively optimized a lead vemurafenib-based PROTAC, SJF-0628, by coupling vemurafenib to a ligand for the von Hippel Lindau (VHL) E3 ligase using a rigid piperazine linker (Fig. 1a). In addition, we synthesized a degradation-incompetent control, SJF-0661, by inverting the stereocenter of the critical hydroxylproline group in the VHL ligand ${ }^{22,35}$. In NIH3T3 cells expressing doxycycline-inducible ${ }^{14,15}$, V5-tagged BRAFWT ${ }^{\mathrm{WT}}$, Class 1,2 , or 3 BRAF mutations, SJF-0628 caused a dose-dependent decrease in the expression of all tested BRAF mutants, but spared BRAFWT, ARAF, and CRAF (Fig. 1b, Supplementary Fig. 1c). Mutant selectivity was also observed in 293 T-Rex cells expressing HAtagged BRAF isoforms (Supplementary Fig. 1d).

To confirm these findings, we evaluated the ability of SJF-0628 to degrade endogenously expressed BRAF mutants in cancer cells. SJF-0628 treatment of SK-MEL-28 cells (homozygous BRAFV600E) resulted in a $\mathrm{DC}_{50}$ (half-maximal degradation) value of $6.8 \mathrm{nM}$ and $D_{\text {MAX }}$ (percent of maximal degradation) of $>95 \%$ (Fig. 1c); similar results were seen in A375 cells (homozygous BRAFV600E) (Supplementary Fig. 2a). In SK-MEL-239 cells (heterozygous BRAF $^{\mathrm{V} 600 \mathrm{E}}$ ), minimal BRAF degradation was observed, likely due to residual BRAFWT although, there is a similarly sustained decrease in MAPK signaling (Supplementary Fig. 2b).

Treatment with $10 \mathrm{nM}$ SJF-0628 caused maximal inhibition of MEK and ERK phosphorylation in SK-MEL-28 cells (Fig. 1c). As expected, while the epimer control, SJF-0661, did not decrease BRAF protein levels (Supplementary Fig. 2c), inhibition of MEK and ERK phosphorylation by this vemurafenib-based molecule was nonetheless observed. However, maximal suppression of $\mathrm{p}$-ERK required $100 \mathrm{nM}$ of SJF-0661, affirming a 10-fold increase in potency for the PROTAC from targeting both the enzymatic and non-enzymatic roles of BRAF (Fig. 1d). SJF-0628 induced near complete BRAFV600E degradation within $4 \mathrm{~h}$ (Fig. 1e, Supplementary Fig. 2d), and $\mathrm{BRAF}^{\mathrm{V} 600 \mathrm{E}}$ degradation and $\mathrm{p}$-ERK inhibition was sustained for up to $72 \mathrm{~h}$ (Supplementary Fig. 2e). A wash-out experiment of SJF-0628 after a $24 \mathrm{~h}$ treatment showed $30 \%$ recovery of BRAF levels and MAPK phosphorylation after $24 \mathrm{~h}$, confirming the long acting and possibly catalytic effect of PROTACs (Supplementary Fig. 2f). BRAFV $600 \mathrm{E}$ degradation was prevented when cells were pre-treated with epoxomicin (proteasome inhibitor) ${ }^{36}$, MLN-4924 (neddylation

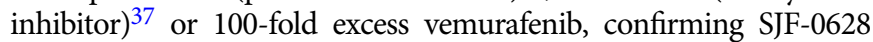
mediated protein loss is consistent with a PROTAC mechanism of action (Supplementary Fig. 2g). Treatment of VHL ligand alone did not affect MAPK phosphorylation, showing that the effect of the PROTAC is primarily due to degradation of BRAF (Supplementary Fig. 2h).

One clinically observed acquired resistance mechanism to vemurafenib is the aberrantly spliced BRAF mRNA transcript encoding an N-terminally truncated isoform that signals as a constitutive dimer (BRAF-p61 $\left.{ }^{\mathrm{V} 600 \mathrm{E}}\right)^{38}$. In SK-MEL-239 C4 cells (BRAFWT/BRAF-p61 $\left.^{\mathrm{V} 600 \mathrm{E}}\right)^{38}$, SJF-0628 induced the degradation of the p61 dimer with a $\mathrm{DC}_{50}$ of $72 \mathrm{nM}$ and $D_{\mathrm{MAX}}>80 \%$, while notably sparing BRAF ${ }^{\mathrm{WT}}$ and CRAF (Fig. 1f). Similar results were seen in HCC-364 vrlcells (BRAFWT/BRAF-p61 $\left.{ }^{\mathrm{V} 600 \mathrm{E}}\right)^{39}\left(\mathrm{DC}_{50}\right.$ of 
a

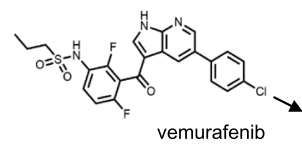

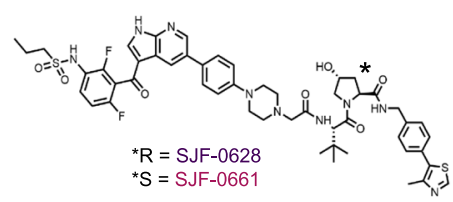

d

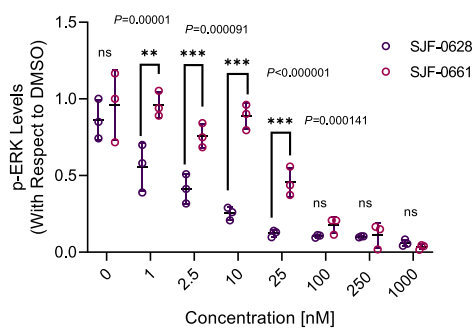

e
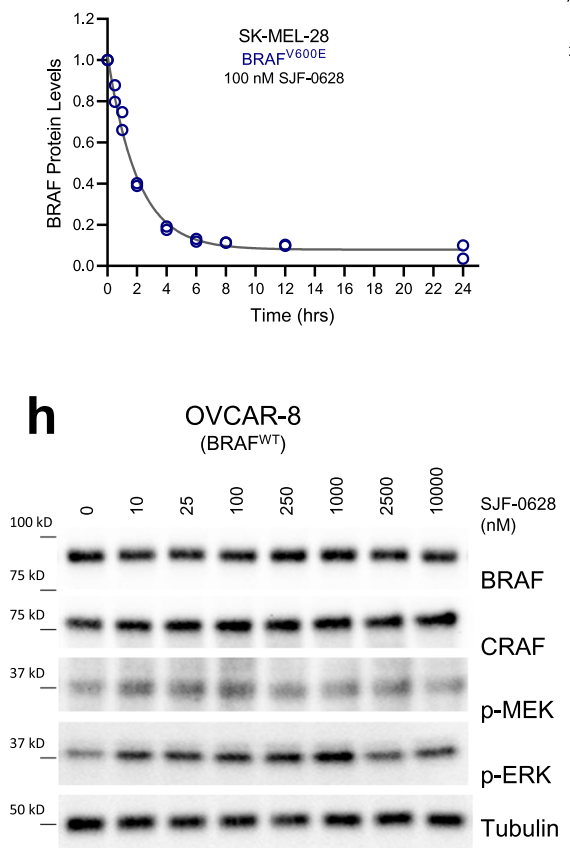

b

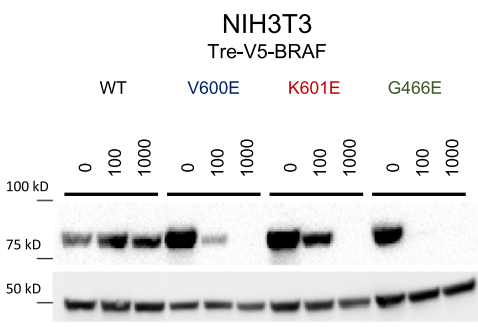

$\mathbf{f}$

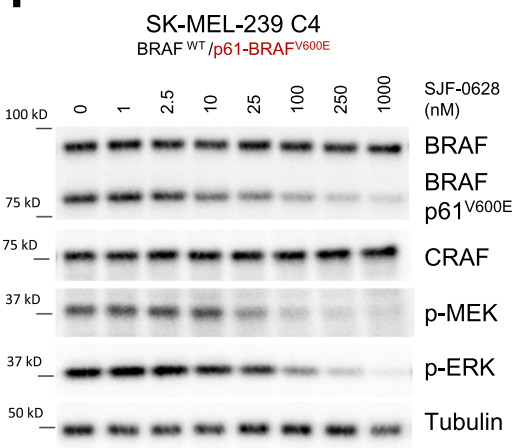

C

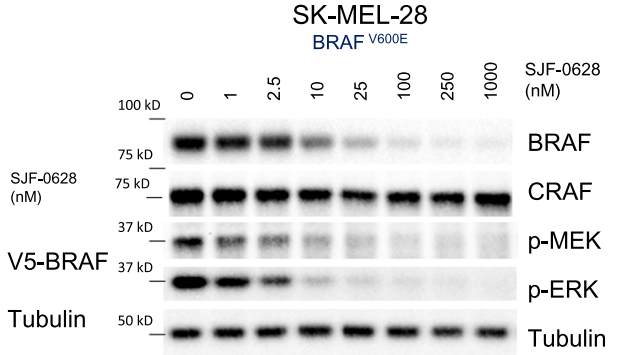

\section{g}

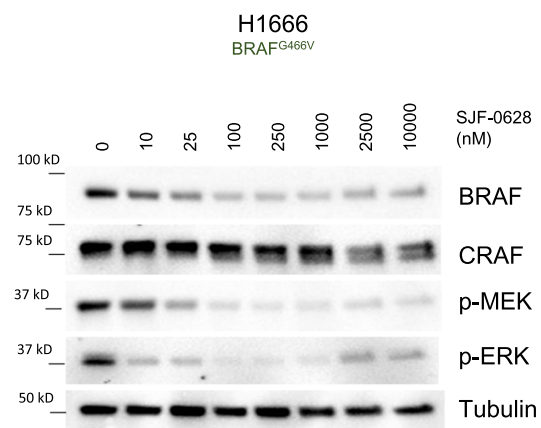

Fig. 1 Vemurafenib-based PROTAC SJF-0628 induces degradation of mutant BRAF. a Chemical structure of vemurafenib and BRAF targeting PROTAC, SJF-0628, and its epimer, SJF-0661. SJF-0628 is composed of vemurafenib, a short piperazine-based linker, and a VHL recruiting ligand. SJF-0661 has an identical warhead and linker as SJF-0628 but contains an inverted hydroxyl group in the VHL ligand and is therefore unable to engage VHL to induce ubiquitination. b Inducible NIH3T3 cells expressing indicated V5-BRAF constructs (doxycycline 100-200 ng/mL, $24 \mathrm{~h}$ ) treated with increasing amounts of SJF-0628. c SK-MEL-28 cells (homozygous BRAF ${ }^{600 E}$ ) treated with indicated amounts of SJF-0628 induced BRAF degradation and suppression of MEK and ERK phosphorylation. d Quantitation of ERK inhibition in SK-MEL-28 cells treated with SJF-0628 or SJF-0661 (mean \pm SD, $n=3$ biologically independent samples) $P$ value calculated by multiple unpaired $t$-tests. e Quantitation of SJF-0628 treatment time course (100 nM) at indicated times in SKMEL-28 cells shows maximal degradation within $4 \mathrm{~h}$ ( $n=2$ biologically independent samples). $\mathbf{f}$ SJF-0628 induces selective degradation of $\mathrm{p} 61-\mathrm{BRAF} \mathrm{V} 600 \mathrm{E}$ mutant and inhibits MEK and ERK phosphorylation but spares BRAFWT and CRAF in SK-MEL-239-C4 cells. g H1666 (heterozygous BRAFG466V) treated with SJF-0628 shows BRAF degradation, but incomplete suppression of ERK signaling. h BRAFWT is spared by SJF-0628 in OVCAR-8 cells but induces slight activation of ERK phosphorylation. $\mathbf{i}$ Covalent inhibition of KRAS G12C by MRTX849 in H23 cells hinders PROTAC induced BRAFWT degradation ( $n=3$ biologically independent samples). $\mathbf{j}$ Quantification of $\mathbf{1 i}$ (mean $\pm S D, n=3$ biologically independent samples). $P$ value calculated by one-way ANOVA. Source data are provided as a Source Data file.

$\left.147 \mathrm{nM}, D_{\mathrm{MAX}}>90 \%\right)$ and 293 T-Rex cells overexpressing HABRAF-p61 V600E (Supplementary Fig. 3a, b). In SK-MEL-246 cancer cells ${ }^{40}$ (Class 2, BRAFG469A), SJF-0628 induced dosedependent degradation of BRAF $\left(\mathrm{DC}_{50}=15 \mathrm{nM}, D_{\mathrm{MAX}}>95 \%\right)$ and concomitant inhibition of ERK phosphorylation while CRAF is slightly stabilized (Supplementary Fig. 3c).

Class 3 BRAF mutants are kinase dead or hypoactive and are frequently observed in NSCLC ${ }^{15,41}$. Unlike inhibitors, PROTACs 
offer a way to target the non-enzymatic/scaffolding role of these BRAF mutants by promoting their degradation. Treatment of NSCLC cell lines H1666 and CAL-12-T cells (Class 3, BRAFG466V; heterozygous and homozygous, respectively) with SJF-0628 caused a dose-dependent loss in BRAF protein levels in both cell lines (CAL-12T: $\left.\mathrm{DC}_{50}=23 \mathrm{nM}, D_{\mathrm{MAX}}>90 \%\right)(\mathrm{H} 1666$ cells: $\mathrm{DC}_{50}=29 \mathrm{nM}, D_{\mathrm{MAX}}>80 \%$ ) (Fig. 1g, Supplementary Fig. 3d) as well as substantial p-ERK inhibition but showed slight stabilization at SJF-0628 concentrations higher than $1 \mu \mathrm{M}$.

BRAFWT activation via upstream effectors sensitizes it to SJF0628 induced degradation at high concentrations. We next asked whether BRAFWT is spared from SJF-0628-induced degradation in cancer cells as observed in the NIH3T3 overexpression system. In the ovarian carcinoma cell line OVCAR-8, SJF-0628 similarly induced minor BRAFWT degradation and a slight induction of p-ERK (Fig. 1h). Given that activation shifts BRAFWT from a closed (extended contact with $\mathrm{N}$ terminus) to an open conformation ${ }^{42-44}$, we sought to determine whether this conformational change can affect BRAFWT susceptibility to SJF0628 in cells with either amplified receptor tyrosine kinases (RTKs) or mutant RAS. In contrast to cells lacking constitutive upstream signaling, we observed $\sim 30 \%$ degradation of BRAFWT in A-431 cells (HER1 amplification) and $\sim 50 \%$ degradation of BRAFWT in SK-BR-3 cells (HER2 amplification) at PROTAC concentrations greater than $1 \mu \mathrm{M}$ (Supplementary Fig. 4a, 8c-left panel). Despite the limited BRAFWT degradation, we still observe paradoxical activation of MAPK signaling (increased p-ERK levels), likely due to PROTAC engagement of residual BRAFWT and/or CRAF. Furthermore, addition of EGF to stimulate the MAPK pathway in OVCAR8 cells sensitized BRAFWT to PROTAC-induced degradation (Supplementary Fig. 4b, c). In cells with mutant RAS (HCT-116, NCI-H23, and SK-MEL-30), the PROTAC also reduced BRAFWT protein levels by $50-60 \%$ and caused ERK activation (Supplementary Fig. 4d-f). In H23 cells, reduction in BRAF expression was not accompanied by a change in its mRNA, suggesting that its degradation is induced by SJF-0628 (Supplementary Fig. 4g).

Our results suggest that the activated conformation of BRAF may be sensitized to PROTAC-induced degradation. Accordingly, we tested whether inhibition of upstream signaling in these cells, which would reduce BRAF activation, would also reduce the effects of the PROTAC. In SK-BR-3 cells, the HER2/EGFR kinase inhibitor lapatinib (2-hour pre-treatment) reduced SJF-0628-dependent BRAFWT degradation from $48 \%$ to $10 \%$ (Supplementary Fig. $4 \mathrm{~h}$, i). Similarly, in NCI-H23 cells, pre-treatment with the K-RAS G12C inhibitor, MRTX849, for $2 \mathrm{~h}$ also desensitized BRAFWT to the PROTAC: from 50\% BRAFWT degradation in cells treated with the PROTAC alone to $20 \%$ degradation in the MRTX849 pre-treated cells (Fig. 1i, j). Thus, sensitivity of BRAFWT to SJF-0628-mediated degradation is associated with activation of its upstream effectors.

Exploration of SJF-0628 mutant selectivity shows BRAFWT is unable to form a stable ternary complex in cellulo. The selectivity of SJF-0628 for mutant BRAF over BRAFWT suggests that it may have little on-target toxicity and therefore a wide therapeutic index in patients. However, the mechanism of this selectivity is not clear since vemurafenib binds BRAFWT as well as BRAF mutants. During lead optimization, several vemurafenib-based PROTACs were synthesized with varied linker lengths and composition. Similar to SJF-0628, these PROTACs selectively induced degradation of mutant BRAF (Supplementary Fig. 5a, b). Furthermore, during the preparation of this manuscript, two groups published cereblon-based PROTACs, which incorporated vemurafenib or BI882370, that induces BRAFV600E degradation and also spared BRAFWT 45,46. As this phenomenon appears to hold true for multiple BRAF-targeting PROTACs, we explored the mechanism that underlies the observed selectivity.

We evaluated the distinct mechanistic steps that PROTACs undertake to induce degradation: target engagement, ternary complex formation (target protein: PROTAC: E3 ligase) and target ubiquitination. In a radioactive in vitro assay of purified RAF kinase activity, SJF-0628 potently inhibited both BRAFWT $\left(\mathrm{IC}_{50}=5.8 \mathrm{nM}\right)$ and BRAFV600E $\left(\mathrm{IC}_{50}=1.87 \mathrm{nM}\right)$. (Fig. 2a; Table 1) Generally, Class 2 mutants bound SJF-0628 with weaker affinity, but nevertheless are successfully degraded in cells; Class 3 mutants were not tested due to their inherent weak kinase activity. Furthermore, SJF-0628 induces paradoxical activation of MAPK signaling, showing that BRAFWT engagement is also achieved in cells. Thus, binary binding is not the basis of isoform degradation selectivity by SJF-0628.

To determine whether differences in ternary complex formation explain the differential degradation observed, we performed a pulldown experiment by immobilizing recombinant GST-tagged VHL/Elongin B/Elongin C (VBC) on glutathione-sepharose and incubating the beads with purified full-length BRAF and increasing amounts of PROTAC, with the goal of detecting a VHL: PROTAC: BRAF trimer. Indeed, there was a dose-dependent increase of $\mathrm{BRAF}^{\mathrm{WT}}$ and BRAFV600E complexed with the VBC at comparable levels (Fig. 2b, c). In fact, BRAFWT appeared to form a stronger ternary complex than BRAFV600E. Hence the innate capacity to form a trimer therefore does not contribute to BRAF isoform selectivity of degradation. However, mutant and BRAFWT adopt different conformations and form unique complexes within cells $^{4,47}$. Thus, we hypothesized that BRAF cellular conformations or associated proteins may affect trimer formation. To investigate this, we performed pull-down assays using NIH3T3 cell lysates expressing V5-BRAFWT or V5-BRAFV600E. Interestingly, we found that while BRAFWT forms a ternary complex with VHL in this lysate-based assay, this occurs to a lesser extent than it does for $\mathrm{BRAF}^{2600 \mathrm{E}}$ ( $\sim 3$ fold greater than BRAFWT at $5000 \mathrm{nM}$ ), as well as for BRAFK601E and BRAFG466E (Fig. 2d, e and Supplementary Fig. 6a). This result suggested that the in vitro trimer formation system using purified recombinant BRAF may not fully recapitulate PROTAC-induced complex formation in cells.

Therefore, we sought to further examine ternary complex formation in cells. We treated NIH3T3 cells with SJF-0628 for $1 \mathrm{~h}$ (to minimize degradation) and pulled down V5-BRAF and any associated VHL E3 ligase components. While SJF-0628 induced an interaction of all three mutant BRAF classes with Cullin 2, the E3 adaptor of VHL, it did not promote BRAFWT trimer formation (Fig. 2f). At higher concentrations, we observe some ternary complex formation with BRAFWT, but much less than that seen with mutant BRAF (Supplementary Fig. 6b, c). To further examine PROTAC induced proximity in intact cells, we employed the NanoLuc bioluminescent resonance energy transfer (NanoBRET) system comprised of transiently co-expressed C-terminal tagged NanoLuc-BRAF (donor) isoforms and HaloTAG-VHL labeled with a HaloTAG NanoBRET 618 ligand (acceptor). In this system, while SJF-0628 caused an increase in BRET signal in cells expressing $\mathrm{BRAF} \mathrm{F}^{\mathrm{V} 600 \mathrm{E}}$, there was no observed increase in cells expressing BRAFWT at concentrations as high as $10 \mu \mathrm{M}$ (Fig. 2g). We did not observe an increase in BRET ratio in the absence of BRAF or VHL, confirming that the BRET signal observed is indeed due to ternary complex formation (Supplementary Fig. 6d). Furthermore, while all three mutant classes are ubiquitinated in cells, BRAFWT is not (Fig. 2h, Supplementary Fig. 6e, f). Overall, these studies show that BRAFWT weakly associates with the E3 ligase complex in the cellular milieu, and this leads to minimal ubiquitination and degradation as compared to mutant BRAF; hence, the mutant selectivity of the PROTAC. 


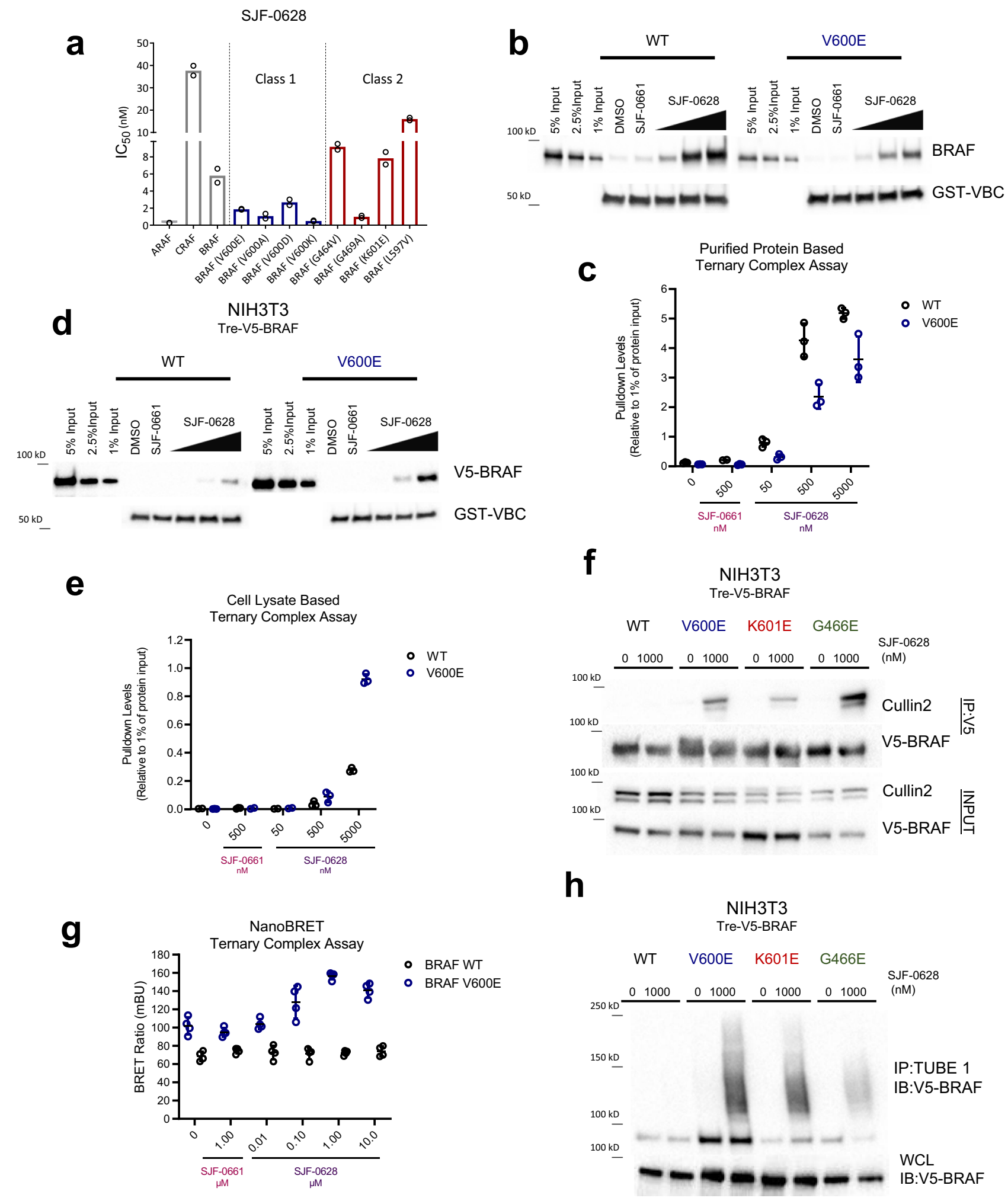

Relief of negative feedback sensitizes BRAFWT to SJF-0628 induced degradation. Our results suggest that BRAFWT conformation and complex heavily influences its degradability. Therefore, we directly interrogated how these properties affect the ability to induce BRAFWT degradation. Studies have shown that MEK inhibition potentiates the activated state of RAF by attenuating feedback inhibition from downstream effectors to stabilize/increase RAF dimerization and association with
RAS $^{48,49}$. Therefore, we hypothesized that MEK inhibition would allow for enhanced BRAFWT degradation.

To test this, we pre-treated NIH3T3 cells with the allosteric MEK inhibitor, trametinib, followed by increasing doses of SJF0628. Interestingly, trametinib addition caused dose-dependent PROTAC-induced BRAFWT degradation, as well as increased MEK phosphorylation (Fig. 3a, Supplementary Fig. 7a); trametinib effectiveness was confirmed by the minimal ERK 


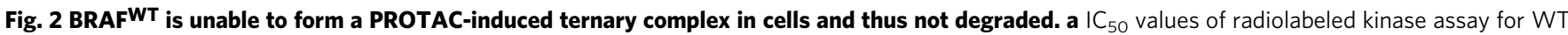
RAF and Class 1 and 2 BRAF mutants (mean, $n=2$ biologically independent experiments). Plotted values shown in Table 1. $\mathbf{b}$ Purified protein ternary complex assay. GST-VBC (VHL, Elongin B, Elongin C) is immobilized on glutathione beads and incubated with DMSO, SJF-0661 (500 nM) or increasing concentrations of SJF-0628 and purified full length-BRAF to observe VBC:PROTAC:BRAF ternary complex. c Quantification of $\mathbf{2 b}$ with respect to $1 \%$ input (mean \pm SD, $n=3$ biologically independent samples). Replicates shown in source data; WT = black circles, V600E $=$ blue circles. $\mathbf{d}$ Cell lysate based ternary complex assay (as described in b) but using NIH3T3 cell lysates (doxycycline $800 \mathrm{ng} / \mathrm{mL}$ ) containing V5-BRAFWT or V5-BRAFV600E as input. e Quantification of $\mathbf{2 d}$ with respect to $1 \%$ input (mean $\pm S D, n=3$ biologically independent samples). Replicates shown in source data. WT $=$ black circles, $\mathrm{V} 600 \mathrm{E}=$ blue circles. $\mathbf{f} \mathrm{NIH} 3 \mathrm{~T} 3$ cells expressing indicated V5-BRAF treated with DMSO or $1 \mu \mathrm{M} \mathrm{SJF-0628}$ for 1-hour followed by immunoprecipitation of V5-BRAF. g NanoBRET ternary complex assay. HEK293T cells ectopically expressing NanoLuc-BRAF(donor) and HaloTag-VHL covalently labeled with a HaloTag 618 ligand (acceptor) were treated with DMSO, epimer SJF-0661(1 $\mu \mathrm{M}$ ) or indicated concentration of SJF-0628 for $3 \mathrm{~h}$. Data represented as BRET ratio (mean $\pm S D, n=4$ biologically independent experiments); WT = black circles, V600E = blue circles. $\mathbf{h}$ Tandem Ubiquitin Binding Entities 1 (TUBE1) pull down of tetra-ubiquitinated proteins in NIH3T3 cells expressing indicated V5-BRAF after 1-hour treatment with vehicle or SJF-0628. Immunoblotted for V5-BRAF. Source data are provided as a Source Data file.

\begin{tabular}{|c|c|}
\hline Kinase: & SJF-0628 IC 50 (nM) \\
\hline ARAF & 0.27 \\
\hline CRAF & 37.6 \\
\hline BRAF & 5.80 \\
\hline BRAF (V600E) & 1.87 \\
\hline BRAF (V600A) & 1.06 \\
\hline BRAF (V600D) & 2.68 \\
\hline BRAF (V600K) & 0.49 \\
\hline BRAF (G464V) & 9.18 \\
\hline BRAF (G469A) & 0.98 \\
\hline BRAF (K601E) & 7.84 \\
\hline BRAF (L597V) & 16.0 \\
\hline
\end{tabular}

phosphorylation observed. Trametinib pre-treatment did not prevent $\mathrm{BRAF}^{\mathrm{V} 600 \mathrm{E}}$ degradation, nor did it promote epimerinduced degradation of $\mathrm{BRAF}^{\mathrm{V} 600 \mathrm{E}}$ or $\mathrm{BRAF}^{\mathrm{WT}}$ in NIH3T3 cells (Supplementary Fig. 7b, c). SJF-0628 also induced BRAFWT degradation in NIH3T3 cells that were pre-treated with cobimetinib - a second, structurally distinct allosteric MEK inhibitor (Fig. 3a). In OVCAR-8 cells, pre-treatment with cobimetinib or trametinib also enabled PROTAC-induced $\mathrm{BRAF}^{\mathrm{WT}}$ degradation while increasing MEK and CRAF phosphorylation (Fig. 3b). Cobimetinib pre-treatment stimulated MEK phosphorylation within $30 \mathrm{~min}$, and enabled PROTACinduced degradation of BRAFWT within $4 \mathrm{~h}$, with complete degradation observed after $12 \mathrm{~h}$ (Supplementary Fig. 7d). No changes in BRAFWT mRNA levels were observed (Supplementary Fig. 7e) confirming that BRAFWT downregulation by SJF-0628 in the presence of cobimetinib is, indeed, post-translational. Moreover, we observed markedly increased trimer formation in cell lysates pre-treated with cobimetinib (Fig. 3c). In addition, MEK inhibitor-pretreated cells generated a 4-fold increase in PROTAC-induced Cullin-2 association with BRAFWT (Fig. 3d) and increased SJF-0628-dependent BRAFWT ubiquitination (Fig. 3e) showing that the BRAFWT degradation observed occurred via a PROTAC mechanism of action. These data, taken together, support our hypothesis that the activated conformation drives the ability of the PROTAC to degrade BRAFWT.

To rule out other aspects of MEK inhibition that may promote BRAFWT $^{W}$ degradation by SJF-0628, we undertook a series of pharmacological studies. In addition to inhibiting negative feedback and increasing BRAF activity, cobimetinib and trametinib have also been shown to decrease BRAFWT association with MEK $^{50}$. As such, MEK might hinder BRAFWT ternary complex formation with SJF-0628 and VHL, preventing degradation of $\mathrm{BRAF}^{\mathrm{WT}}$. To investigate this, we pre-treated cells with an early generation MEK inhibitor, PD0325901, known to stabilize the BRAF:MEK complex ${ }^{51}$ (Supplementary Fig. 8a). However, cells pretreated with PD0325901 also enabled PROTAC-mediated BRAFWT degradation ( $\sim 80 \%$ degradation at $1 \mu \mathrm{M})$ (Fig. 3f). We hypothesized that if relief of MAPK negative feedback promotes BRAFWT degradation, ERK inhibition would do the same. As predicted, pretreatment with the selective ERK inhibitor SCH772984 52 at $1 \mu \mathrm{M}$ enabled $\sim 90 \%$ degradation of $\mathrm{BRAF}^{\mathrm{WT}}$ by the PROTAC (Fig. 3f). SCH772984 also does not disrupt BRAF-MEK association, further demonstrating that the presence of MEK does not affect BRAFWT degradation (Supplementary Fig. 8a).

GDC-0623 is an allosteric MEK inhibitor which binds MEK in a manner that sequesters BRAF and hinders dimerization with itself or CRAF (Supplementary Fig. 8a) and membrane localization ${ }^{50,53}$. Therefore, treatment with GDC-0623 dampens relief of feedback induced signaling on RAF kinase activity. We hypothesized that if the BRAFWT conformation induced by the previously tested $\mathrm{MEK} / \mathrm{ERK}$ inhibitors is primarily responsible for enabling the kinase's degradation by the PROTAC, then GDC-0623 would enable significantly less degradation. As expected, GDC-0623 pretreatment permitted only minimal PROTAC-dependent degradation of $B R A F^{W T}$ - far less than was seen in parallel treatment with cobimetinib (Fig. 3g, Supplementary Fig. 8b, c). These results further show that SJF-0628 selectively induces degradation of $B R A F^{W T}$ in its active conformation. Indeed, studies such as Rock et al. show that all three mutant BRAF classes (including kinase dead mutations) favor an open, active conformation ${ }^{54}$. Thus, by stimulating BRAFWT activity (e.g., RTK upregulation, RAS mutations, relief of negative feedback), we promote an open conformation which is susceptible to increased ternary complex formation and therefore degradation.

SJF-0628 successfully inhibits cell growth in mutant-BRAF driven cancer cells. Next, we compared the effects on cell growth of inhibiting both the enzymatic and scaffolding roles of BRAF using SJF-0628, with those of an ATP-competitive inhibitor that targets only its catalytic function (vemurafenib and degradationincompetent epimer, SJF-0661). In SK-MEL-28 cells (Class 1, $\mathrm{BRAF}^{\mathrm{V} 600 \mathrm{E}}$ ), vemurafenib and SJF-0661 inhibited cell growth with an $\mathrm{EC}_{50}$ of $215 \pm 1.09 \mathrm{nM}$ and $243 \pm 1.09 \mathrm{nM}$, respectively, while SJF-0628 showed an $\mathrm{EC}_{50}$ of $37 \pm 1.2 \mathrm{nM}$ (Fig. 4a). The 6-fold increase in potency of SJF-0628 occurred despite the compounds having similar in vitro binding (vemurafenib $=27 \mathrm{nM}$, SJF-0628 $=$ $39 \mathrm{nM}$, SJF-0661 $=64 \mathrm{nM}$ ) (Supplementary Fig. 9a, Supplementary Table 2). In SK-MEL-239 C4 cells (BRAFWT/BRAF-p61 ${ }^{\mathrm{V} 600 \mathrm{E}}$ ), while vemurafenib and SJF-0661 had a minimal effect, SJF-0628 induced $\sim 80 \%$ decrease in cell growth with an $\mathrm{EC}_{50}$ of $218 \mathrm{nM} \pm$ 1.06 (Fig. 4b). This result shows that targeted degradation can be used to overcome acquired resistance to BRAF inhibitor-based 

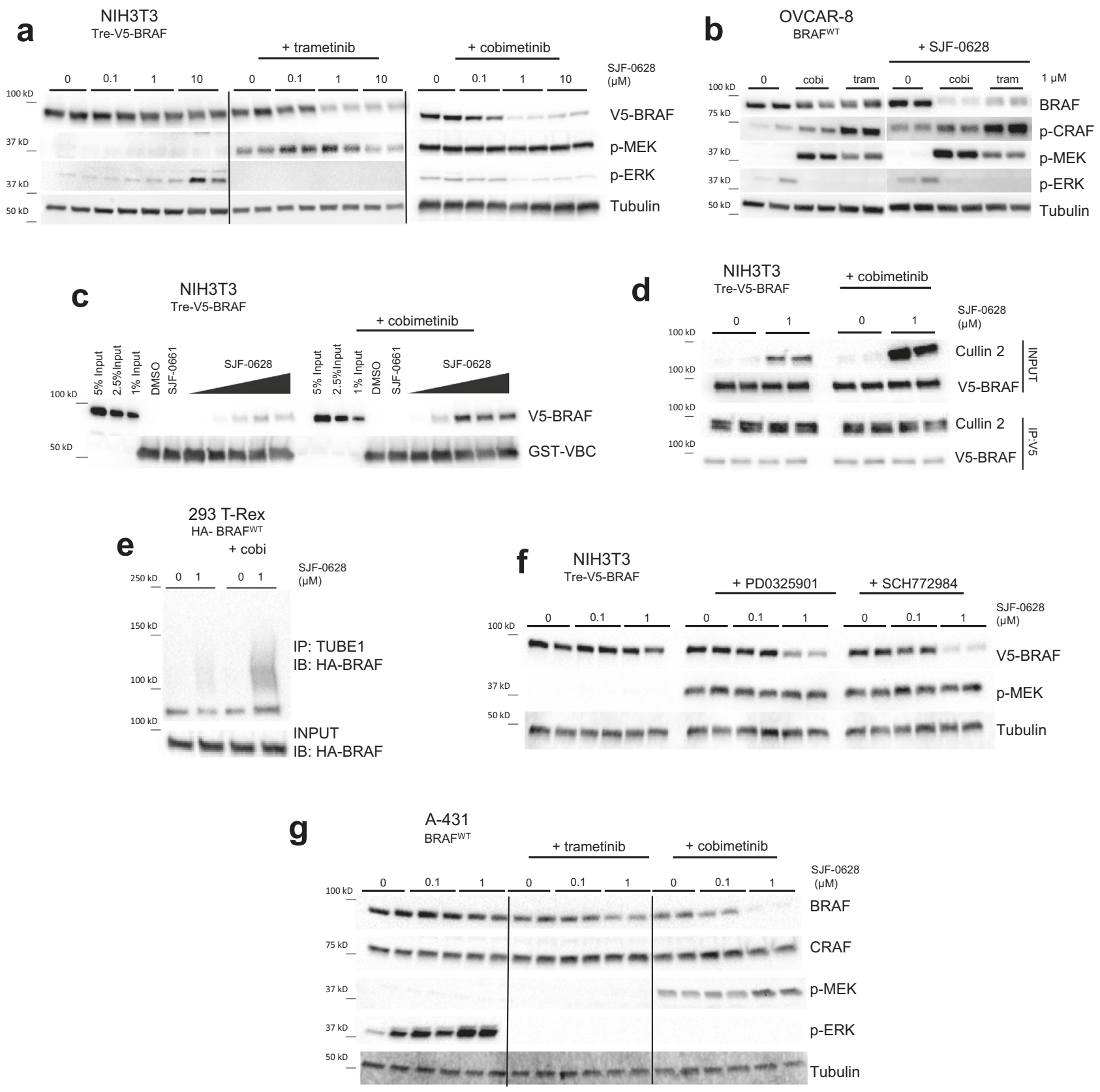

Fig. 3 BRAF-activating MEK inhibitors also sensitize BRAF to PROTAC-induced ubiquitination and degradation. a NIH3T3 cells with trametinib ( $1 \mu \mathrm{M}$, $5 \mathrm{~h}$ ) or cobimetinib ( $500 \mathrm{nM}, 3 \mathrm{~h}$ ) pre-treatment subsequently treated with increasing amounts of SJF-0628 (20 h) promote degradation of BRAFWT and show a marked increase in p-MEK ( $n=2$ biologically independent samples). b OVCAR8 cells pre-treated with cobimetinib and trametinib ( $1 \mu \mathrm{M}, 2 \mathrm{~h}$ ) promote MEK and CRAF phosphorylation as well as BRAF degradation in the presence of SJF-0628 ( $n=2$ biologically independent samples). $\mathbf{c}$ Cell lysatebased ternary complex assay shown in $2 \mathrm{c}$ but using NIH3T3 lysates expressing BRAFWT and pre-treated with DMSO or $1 \mu \mathrm{M}$ cobimetinib for $3 \mathrm{~h}$. Cobimetinib pre-treatment promotes ternary complex formation. d V5-BRAF immunoprecipitation in NIH3T3 cells pre-treated with $1 \mu \mathrm{M}$ of cobimetinib ( $2 \mathrm{~h}$ ) followed by treatment of SJF-0628 for $2.5 \mathrm{~h}$. e TUBE1 pulldown in 293 T-Rex cells stably expressing HA- BRAFWT treated with cobimetinib (cobi) $(2 \mathrm{~h}, 1 \mu \mathrm{M})$ and subsequently treated with SJF-0628 (2 h). f NIH3T3 cells pre-treated with $1 \mu \mathrm{M}$ PD0325901 (MEK inhibitor) or SCH772984 (ERK inhibitor) for $3 \mathrm{~h}$ followed by treatment with indicated amount of SJF-0628 for $20 \mathrm{~h}$. $\mathbf{g}$ A431 cells pre-treated with GDC-0623 and cobimetinib (500 nM for $3 \mathrm{~h}$ ) then treated with SJF-0628 for $20 \mathrm{~h}$. Source data are provided as a Source Data file.

therapies. A 5-day treatment in SK-MEL-246 cells (Class 2, BRAFG469A) SJF-0628 efficaciously inhibited cell growth with and $\mathrm{EC}_{50}$ of $45 \pm 1.11 \mathrm{nM}$ and the epimer showed and an $\mathrm{EC}_{50} 278 \pm$ $1.07 \mathrm{nM}$. However, vemurafenib caused some inhibition of SKMEL-246 cellular growth at concentrations above $1 \mu \mathrm{M}$ which was not sustained at $10 \mu \mathrm{M}$ (Fig. 4c). In H1666 cells (Class 3, $\left.\mathrm{BRAF}^{\mathrm{G} 466 \mathrm{~V}}\right)$, SJF-0628 was able to induce $65 \%$ cell growth inhibition while vemurafenib showed less than 50\% (Fig. 4d). Despite causing $>70 \%$ inhibition of p-ERK (Supplementary Fig. 3d), SJF-0628 showed minimal inhibition of cell growth in CAL-12-T cells (Class 3, BRAFG466V) (Fig. 4e).

SJF-0628 causes potent degradation of all BRAF mutant classes and has minor effects on WT RAF. This result suggests that the PROTAC will be effective in treating tumors driven by these 

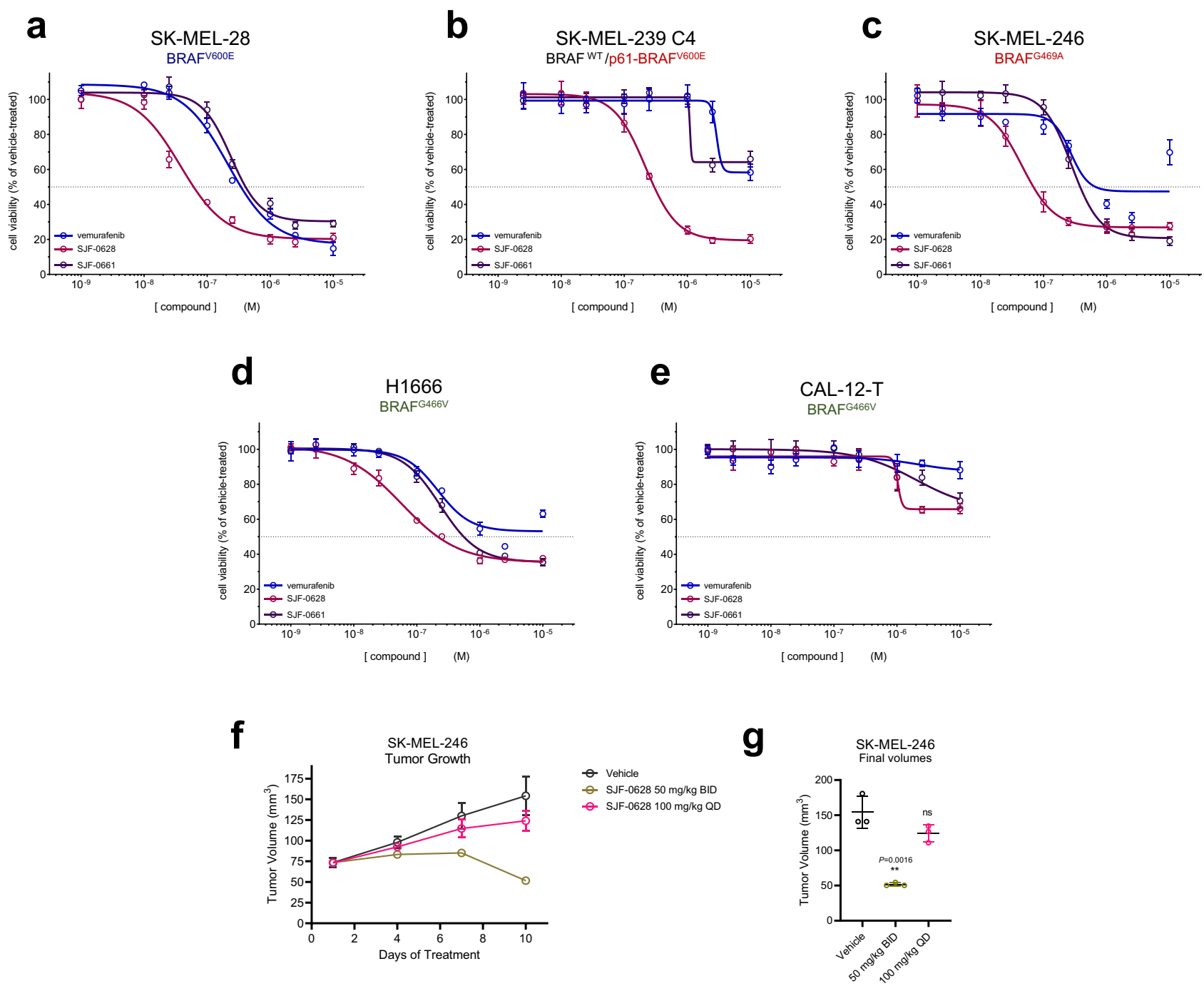

[SJF-0628]

Fig. 4 SJF-0628 outperforms vemurafenib in inhibiting growth of cell lines expressing mutant BRAF. a Cell proliferation assay in SK-MEL-28 cells treated with increasing amounts of vemurafenib, SJF-0628, or SJF-0661 for 3 days (mean \pm SD, $n=3$ biologically independent samples). $\mathrm{EC}_{50}=215 \pm 1.09$ $\mathrm{nM}, 37 \pm 1.2 \mathrm{nM}$, and $243 \pm 1.09 \mathrm{nM}$, respectively; vemurafenib = blue, SJF-0628 = burgundy, SJF-0661 = purple. b Cell proliferation assay in vemurafenib resistant SK-MEL-239-C4 cells treated with increasing amounts vemurafenib, SJF-0628, or SJF-0661 for 5 days (mean \pm SD, $n=3$ biologically independent samples); vemurafenib = blue, SJF-0628 = burgundy, SJF-0661 = purple. c Cell proliferation assay in SK-MEL-246 (Class 2) cells treated with increasing amounts vemurafenib, SJF-0628, or SJF-0661 for 5 days (mean \pm SD, $n=3$ biologically independent samples); vemurafenib $=$ blue, SJF-0628 $=$ burgundy, SJF-0661 = purple. $\mathbf{d}$ SJF-0628 EC50 $=218 \mathrm{nM} \pm 1.06 \mathrm{c}, \mathrm{H} 1666$ cells treated with SJF-0628, vemurafenib, or SJF-0661 for 5 days (mean \pm SD, $n=3$ biologically independent samples); vemurafenib = blue, SJF-0628 = burgundy, SJF-0661 = purple. e Treatment of CAL-12-T cells with vemurafenib, SJF0628, or SJF-0661 for 5 days shows minimal effect on cell viability (mean \pm SD, $n=3$ biologically independent samples). $\mathbf{f}$ Results of an efficacy study in SK-MEL-246 tumor xenografts implanted in female athymic mice showing tumor regression with $50 \mathrm{mg} / \mathrm{kg}$ IP twice daily (mean \pm SD, $n=3$ biologically independent animals). $\mathbf{g}$ Scatter plot result of final tumor volumes of SKMEL-246 xenografts treated with SK-MEL-246 (mean \pm SD, $n=3$ biologically independent animals). $P$ value calculated by unpaired $t$-test. Source data are provided as a Source Data file.

mutations with minimal on target toxicity, thus we tested the effects of SJF-0628 in an A375 (BRAFV600E) murine xenograft model. Mice treated with SJF-0628 (three days; $50 \mathrm{mg} / \mathrm{kg}$ or $150 \mathrm{mg} / \mathrm{kg}$ ) showed marked degradation of BRAF in the xenograft at both concentrations $\left(D_{\mathrm{MAX}}>90 \%\right)$ (Supplementary Fig. 9b). Because SJF-0628 successfully induced degradation in vivo, we tested its effect on tumor growth in the SK-MEL-246 melanoma xenograft model (Class 2, BRAFG469A). Strikingly, while once daily $100 \mathrm{mg} / \mathrm{kg}$ treatment showed a minor response, twice daily treatment of $50 \mathrm{mg} / \mathrm{kg}$ induced tumor shrinkage beyond the initial tumor size within 10 days (Fig. 4f, g). We did not observe a significant body weight loss with either dose
(Fig. S9C). Thus, through targeted degradation, SJF-0628 is successfully able to exhibit a significant antitumor effect.

\section{Discussion}

Each BRAF mutation alters how the protein signals in distinct ways, therefore, careful consideration must be taken to select the appropriate inhibitor. Despite tremendous effort to create therapies that target diverse BRAF mutations, all three currently FDAapproved drugs target Class 1 BRAF mutants alone. Furthermore, resistance to current drugs inevitably occurs. Thus, we have developed a vemurafenib-based PROTAC, SJF-0628, that 
outperforms vemurafenib in inhibiting MAPK signaling and

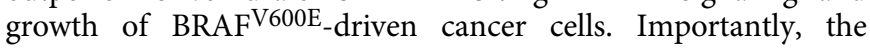
mutant BRAF-targeting PROTACs described here mostly spare WT RAF, thus widening the potential therapeutic window of this new class of anti-tumor drugs.

We find that measuring ternary complexes (BRAF:PROTAC: VHL) in cells or cell lysates to be more predictive of degradation than in vitro studies with purified proteins. Interestingly, similar results were observed by Posternak et al. with cereblon recruiting BRAF-targeting PROTAC ${ }^{45}$. Our in vitro pull-down assays likely contained highly dimerized BRAF in the active conformation, resulting in artificially high levels of ternary complex formation. However, in cells BRAF exists in a closed inactive conformation, which is less conducive to ternary complex formation allowing it to escape degradation. So by promoting its activation, BRAFWT adopts an open conformation, similar to mutant BRAF, and is thus susceptible to SJF-0628-induced degradation. This finding suggests that intracellular protein conformation can affect PROTACinduced degradation. As PROTACs require induced protein-protein interactions to function, it is important to consider protein interactions within the cellular context that might encourage or discourage degradation. Importantly for the development of new PROTACs, we show that we can control induced protein degradation (i.e., BRAF ${ }^{\mathrm{WT}}$ ) without modifying the PROTAC itself but by manipulating its signaling pathway.

While preparing this manuscript, two groups described cereblon-based PROTACs that degrade BRAF $\mathrm{V}^{600 \mathrm{E}}$ but spares $\mathrm{BRAF}^{\mathrm{WT}} 45,46$. Beyond this, we also successfully target vemurafenib-resistant BRAF mutations. This includes mutants that have both acquired (p61 V600E) and intrinsic (Class 2) resistance to vemurafenib. Furthermore, we show that SJF-0628 can be used to successfully target Class 2 mutants in vivo. In addition, we make Class 3 BRAF mutants, which cannot be targeted with traditional small molecule inhibitors, therapeutically accessible through targeted degradation. Indeed, this is another early demonstration of PROTAC induced degradation of a pseudokinase. Thus, using PROTACs, we are able to expand the druggable space to a class of proteins with immense cancer relevance (HER3, ROR2, etc.). In summary, this study demonstrates that the PROTAC technology is an attractive strategy for targeting difficult oncoproteins such as mutant BRAF.

\section{Methods}

PROTAC treatment and immunoblotting. Cells were plated in 6 well dishes $(5 \times$ $10^{5}-8 \times 10^{5}$ cells) and allowed to attach overnight. Cells were treated with SJF-0628 or SJF-0661 for 20-24h (unless otherwise stated). The plates were then placed on ice and washed $1 \mathrm{x}$ with chilled PBS and lysed in buffer containing $25 \mathrm{mM}$ Tris- $\mathrm{HCl}$ [pH 7.4], $0.25 \%$ sodium deoxycholate, $150 \mathrm{mM} \mathrm{NaCl}, 1 \%$ Triton X-100, supplemented with protease inhibitors (1x Roche protease inhibitor cocktail) and phosphatase inhibitors ( $10 \mathrm{mM} \mathrm{NaF}, 1 \mathrm{mM} \mathrm{Na}_{3} \mathrm{OV}_{4}$, and $20 \mathrm{mM} \beta$-glycerophosphate). Lysates were then cleared at $21,000 \mathrm{~g}$ for $10 \mathrm{~min}$ at $4{ }^{\circ} \mathrm{C}$. Protein concentrations of the supernatants were then quantified using a Pierce BCA Protein Assay. 12-40 $\mu \mathrm{g}$ of protein were separated using a gradient (4-20\%) Criterion TGX precast gel and transferred unto a nitrocellulose membrane. The membranes were then blocked in $5 \%$ non-fat milk in TBST (Tris-buffered Saline with Tween 20) for $1 \mathrm{~h}$ before probing with the indicated primary antibody overnight. Membranes were imaged using Bio-Rad Image Lab software using ECL prime detection reagent (GE Healthcare, RPN2232 or ThermoScientific, 34095).

Cell proliferation. Cells (25,000 to 5000) were seeded in 96 well plates and treated with compound for the indicated lengths of time (between 72 and $96 \mathrm{~h}$ ). $2 \mathrm{mg} / \mathrm{ml}$ MTS (Promega Corp., Madison, WI: G5421) and $25 \mu \mathrm{M}$ phenazine methosulfate (Sigma, St. Louis, MO) were combined 19:1 and then added to cells (1 volume combined reagent: 5 volumes medium) and incubated for $1-3 \mathrm{~h}$. Mitochondrial reduction of MTS to the formazan derivative was monitored by measuring the medium's absorbance at $480 \mathrm{~nm}$ using a Perkin Elmer Envision Plate reader.

Protein purification. For the expression of GST-tagged VHL:Elongin B:Elongin C (herein referred to as GST-VBC), wild-type human VHL, Elongin B, and Elongin C were co-expressed in E. coli. BL21(DE3) cells were co-transformed with pBB75-
Elongin C and pGEX4T-2-VHL-rbs-Elongin B and selected in LB medium containing carbenicillin $\left(100 \mu \mathrm{g} \mathrm{mL}^{-1}\right)$ and kanamycin $\left(25 \mu \mathrm{g} \mathrm{mL}^{-1}\right)$ at $37^{\circ} \mathrm{C}$ until OD $600=0.8$, at which point the culture was chilled to $16^{\circ} \mathrm{C}$ and induced with $0.4 \mathrm{mM}$ IPTG for $16 \mathrm{~h}$. Cells were homogenized and lysed using a Branson digital sonifier with lysis buffer composed of $50 \mathrm{mM}$ Tris [pH 8.0], $200 \mathrm{mM} \mathrm{NaCl}, 5 \%$ glycerol, 5 mM DTT containing a $1 \mathrm{X}$ protease inhibitor cocktail tablet (Roche). Clarified cell lysate was applied to glutathione sepharose 4B beads (GE Life Science) and gently rotated for $2 \mathrm{~h}$ at $4{ }^{\circ} \mathrm{C}$. Beads were washed with four column volumes of lysis buffer, followed by four column volumes of elution buffer $(50 \mathrm{mM}$ Tris $\mathrm{pH} 8.0,200 \mathrm{mM}$ $\mathrm{NaCl}, 10 \mathrm{mM}$ glutathione). Eluted protein was assessed for identity and purity via Coomassie staining of sample run on an SDS-PAGE gel and pure elutions were pooled, concentrated, and diluted in ion-exchange buffer A (30 mM Tris pH 8.0, $5 \%$ glycerol, $1 \mathrm{mM}$ DTT) until the salt concentration was $50 \mathrm{mM}$, before loading onto a Mono Q 5/50 GL column (GE Life Sciences). The protein was subjected to a linear gradient of $\mathrm{NaCl}(0-500 \mathrm{mM} \mathrm{NaCl})$ using ion-exchange buffer $\mathrm{B}(30 \mathrm{mM}$ Tris 8.0, $1 \mathrm{M} \mathrm{NaCl}, 5 \%$ glycerol, $1 \mathrm{mM} \mathrm{DTT}$ ). Fractions were then assessed for purity via Coomassie, pooled, concentrated, and run on a Superdex-200 column (GE Life Sciences) using size-exclusion buffer (30 mM Tris pH 8.0, $100 \mathrm{mM} \mathrm{NaCl}$, $10 \%$ glycerol, $1 \mathrm{mM}$ DTT). Pure fractions of GST-VHL were pooled, concentrated to $\sim 5 \mathrm{mg} \mathrm{mL}^{-1}$, aliquoted, and flash-frozen before storing at $-80{ }^{\circ} \mathrm{C}$.

Ternary complex assays. Glutathione sepharose $4 \mathrm{~B}$ was washed twice with water and then blocked for $1 \mathrm{~h}$ at room temperature with 10\% BSA in TBST. The beads were then washed again twice with TBST and once with wash buffer $(50 \mathrm{mM}$ HEPES pH 7.5, $150 \mathrm{mM} \mathrm{NaCl}, 1 \mathrm{mM}$ DTT, $0.01 \%$ NP40, $5 \mathrm{mM} \mathrm{MgCl}_{2}, 10 \%$ glycerol) and then purified GST-VBC was immobilized for $2 \mathrm{~h}$ at $4{ }^{\circ} \mathrm{C}$ at 2.5 pmole per $\mu \mathrm{L}$ of beads. The beads were then washed thrice with wash buffer, resuspended and BRAF WT or V600E protein was added at $500 \mathrm{nM}$ per $50 \mu \mathrm{L}$ reaction with 5 $\mu \mathrm{L}$ of beads. The bead:BRAF mixture was then aliquoted to separate tubes and PROTAC was added at the indicated concentration (PROTACs were intermediately diluted in $50 \%$ DMSO) and this was incubated at $4{ }^{\circ} \mathrm{C}$ for $2 \mathrm{~h}$. The beads were washed 4 times with $1 \mathrm{~mL}$ column of TBST and then eluted with SDS loading buffer.

For experiments in which the input substrate is a whole cell lysate, the sample was prepared as follows: $15 \mathrm{~mm}$ dishes of confluent NIH3T3 cells were doxycycline induced overnight, after which the cells were washed with DPBS and lysed using lysis buffer ( $50 \mathrm{mM}$ Tris- $\mathrm{HCl}, \mathrm{pH} 7.4,015 \mathrm{M} \mathrm{NaCl}, 1 \mathrm{mM}$ EDTA,1\% NP40, 10\% glycerol). The lysate was cleared by centrifugation and then added to the beads as an input substrate, as above. For MEK inhibitor comparison, NIHT3 cells were pretreated with $1 \mu \mathrm{M}$ of cobimetinib for $3 \mathrm{~h}$

NanoBRET ternary complex assay. Full-length BRAF (WT and V600E) was cloned into the pNLF1-C [CMV/Hygro] vector. HEK 293 T cells were seeded into 6 well dishes $(\sim 75 \%$ confluent) and co-transfected with HaloTag-VHL (Promega; $\mathrm{N} 273 \mathrm{~A}$ ) and C-terminal nanoLUC-tagged BRAF. Transfection was performed using a ratio of 1:2 DNA to Lipofectamine 2000 . After $24 \mathrm{~h}, 1 \times 10^{4}$ cells were seeded into white 96 well plates (Costar Cat\# 3610) in Opti-MEM ${ }^{\mathrm{TN}}$ I Reduced Serum Medium supplemented with 4\% FBS and 0.1 mM HaloTag NanoBRET 618 ligand or DMSO. The next day, the cells were pre-treated with $2 \mu \mathrm{M}$ MG-132 for $1 \mathrm{~h}$ then treated with indicated amount of drug for $3 \mathrm{~h}$. 6X NanoBRET Nano-Glo Substrate in Opti-MEM was then added to each well and the plate was read using a Perkin Elmer Envision. Dual filtered luminescence was measured with a $430 \mathrm{~nm}$ (donor, HaloTag NanoBRET ligand) and a $615 \mathrm{~nm}$ filter (acceptor, HaloTag NanoBRET ligand). Background corrected (cells without HaloTag 618 ligand) nanoBRET ratios were calculated to determine intracellular ternary complex formation.

Cellular immunoprecipitation and ubiquitination assay. Doxycycline-induced NIH3T3 cells or 293 T-Rex cells that express indicated BRAF isoform were seeded in $10 \mathrm{~cm}$ dishes overnight. Cells were then treated for $1 \mathrm{~h}$ with PROTAC or DMSO. Cells were then placed on ice, washed with ice-cold 1X PBS, and lysed in $500 \mu \mathrm{L}$ modified 1X lysis buffer (50 mM Tris-HCl, pH 7.4, 0.15 M NaCl, $1 \mathrm{mM}$ EDTA, $1 \%$ NP40, 10\% glycerol) containing $5 \mathrm{mM} \mathrm{1,10-phenanthroline} \mathrm{monohydrate,} 10 \mathrm{mM}$ N-ethylmaleimide, $20 \mu \mathrm{M}$ PR-619, and $1 \mathrm{X}$ protease inhibitor cocktail (Roche). Lysates were spun down at $14,000 \times g$ at $4{ }^{\circ} \mathrm{C}$ for $10 \mathrm{~min}$. Equal amounts of lysate was aliquoted onto $20 \mu \mathrm{L}$ (bed volume) of anti-V5-beads (Sigma, A7345). V5containing proteins were immunoprecipitated from lysates for $2 \mathrm{~h}$ at $4{ }^{\circ} \mathrm{C}$ with gentle rotation, after which samples were spun down at $6000 \times g$ at $4{ }^{\circ} \mathrm{C}$ for $2 \mathrm{~min}$ and the beads were washed 4 times with DPBS. Beads were resuspended in $1 \mathrm{X}$ lithium dodecyl sulfate (LDS) sample buffer containing 5\% 2-mercaptoethanol (ß$\mathrm{ME}$ ). Immunoprecipitated protein was eluted off the beads by heating at $95^{\circ} \mathrm{C}$ for $5 \mathrm{~min}$ and the supernatant was run on an SDS-PAGE gel and evaluated for the presence of immunoprecipitated V5-tagged proteins, as well as Cullin 2. Input refers to the normalized input lysate loaded onto V5-sepharose beads.

TUBE1 immunoprecipitation experiments were carried out exactly as described above, except that equal amount of lysate was loaded onto $20 \mu \mathrm{L}$ TUBE1 agarose (LifeSensors) resin per sample and washed with TBST. 
Radiolabeled kinase assays. Kinase assays were performed by Reaction Biology Corps by their protocol in duplicate using $\mathrm{K}_{\mathrm{m}}$ amounts of ATP calculated for each kinase.

Elisa kinase inhibition assay. Kinase assays were performed by Carna Biosciences by their protocol in duplicate using $\mathrm{K}_{\mathrm{m}}$ amounts of ATP calculated for each kinase.

RT-PCR. Cells were seeded in 12 well plates and treated as described. RNA was isolated with the RNeasy Mini Kit (QIAGEN) and $1 \mu \mathrm{g}$ of total RNA was reverse transcribed using the High Capacity cDNA Reverse Transcription Kit (Applied Biosystems). SYBR Green PCR master mix (Kapa Biosystems) was used for qRTPCR samples were performed and analyzed in triplicate. Relative RNA expression levels were calculated using the ddCt method and normalized to control samples and beta-tubulin was used for normalization. Primers used in this study are included in Supplementary Table 1.

A375 xenograft study. 5 million A375 cells were subcutaneously implanted in female nu/nu mice. Tumors were randomized after a period of 10 days into groups with an average tumor size of $350 \mathrm{~mm}^{3}$, and treated with vehicle (5\% DMSO, $5 \%$ EtOH, and 20\% Solutol HS15 in D5W), 50 mg/kg SJF-0628, or 150 mg/kg SJF-0628 (4 mice per arm) intraperitoneally once a day for 3 days. Mice were sacrificed $8 \mathrm{~h}$ after the final dose. Tissues were harvested, flash frozen, and lysed in $1 \mathrm{X}$ cell lysis buffer (Cell Signaling \#9803) supplemented with protease and phosphatase inhibitors. Harvested tumors were disrupted using metal beads in a Tissuelyser. Homogenates were normalized for protein content and analyzed using SDS-PAGE and Western blotting. All studies were performed in compliance with institutional guidelines under an IACUC approved protocol.

SK-MEL-246 xenograft study. 10 million SK-MEL-246 cells were subcutaneously implanted in female nu/nu mice. The tumor volumes and mice weights were measured twice a week after the implantation. The i.p. treatments with vehicle $(5 \%$ DMSO, 5\% EtOH, and 20\% Solutol HS15 in D5W), $50 \mathrm{mg} / \mathrm{kg}$ (BID) or $100 \mathrm{mg} / \mathrm{kg}$ SJF-0628 (QD), (3 mice/group) were started when the tumor volumes reached an average of $100 \mathrm{~mm}^{3}$. All studies were performed in compliance with institutional guidelines under an Institutional Animal Care and Use Committee (IACUC) approved protocol. Investigators were not blinded when assessing the outcome of the in vivo experiments.

Quantitation and statistical analysis of Western blots. Western blot data was quantified by using the band feature in Image Lab, and values were averaged and analyzed in GraphPad Prism. $\mathrm{DC}_{50}$ and $D_{\mathrm{MAX}}$ values were fitted using a three parameter [inhibitor] versus response and reported directly from the Prism output. Mean \pm SD and unpaired $t$-tests were performed in GraphPad Prism.

Structure visualization. Atomic resolution structures were visualized using Molecular Operating Environment software (MOE-2019.0102).

Statistics and reproducibility. All experiments were performed 2 or more independent times with similar results.

Reporting summary. Further information on research design is available in the Nature Research Reporting Summary linked to this article.

\section{Data availability}

Linker positioning was determined using structure of vemurafenib bound to BRAF (PDB:3OG7). Source data are provided with this paper. All other data are available from the corresponding author on reasonable request. Source data are provided with this paper.

Received: 10 August 2020; Accepted: 14 January 2021;

Published online: 10 February 2021

\section{References}

1. Zhang, W. \& Liu, H. T. MAPK signal pathways in the regulation of cell proliferation in mammalian cells. Cell Res. 12, 9-18 (2002).

2. Roberts, P. J. \& Der, C. J. Targeting the Raf-MEK-ERK mitogen-activated protein kinase cascade for the treatment of cancer. Oncogene 26, 3291-3310 (2007).

3. Kung, J. E. \& Jura, N. Structural basis for the non-catalytic functions of protein kinases. Structure 24, 7-24 (2016).

4. Haling, JacobR. et al. Structure of the BRAF-MEK complex reveals a kinase activity independent role for BRAF in MAPK signaling. Cancer Cell $\mathbf{2 6}$ 402-413 (2014).
5. Garnett, M. J., Rana, S., Paterson, H., Barford, D. \& Marais, R. Wild-type and mutant B-RAF activate C-RAF through distinct mechanisms involving heterodimerization. Mol. Cell 20, 963-969 (2005).

6. Flemming, A. Targeting mutant BRAF in metastatic melanoma. Nat. Rev. Drug Discov. 9, 841-841 (2010).

7. Barras, D. BRAF mutation in colorectal cancer: an update. Biomark. Cancer 7 , 9-12 (2015).

8. Nguyen-Ngoc, T. et al. BRAF alterations as therapeutic targets in non-smallcell lung cancer. J. Thorac. Oncol. 10, P1396-1403 (2015)

9. Tiacci, E. et al. BRAF Mutations in Hairy-Cell Leukemia. New England Journal of Medicine 364, 2305-2315 (2011).

10. Holderfield, M., Deuker, M. M., McCormick, F. \& McMahon, M. Targeting RAF kinases for cancer therapy: BRAF-mutated melanoma and beyond. Nat. Rev. Cancer 14, 455-467 (2014).

11. Dankner, M., Rose, A. A. N., Rajkumar, S., Siegel, P. M. \& Watson, I. R. Classifying BRAF alterations in cancer: new rational therapeutic strategies for actionable mutations. Oncogene 37, 3183-3199 (2018).

12. Forbes, S. A. et al. COSMIC: mining complete cancer genomes in the catalogue of somatic mutations in cancer. Nucleic Acids Res. 39, D945-D950 (2010).

13. Pratilas, C. A. et al. V600EBRAF is associated with disabled feedback inhibition of RAF-MEK signaling and elevated transcriptional output of the pathway. Proc. Natl Acad. Sci. 106, 4519-4524 (2009).

14. Yao, Z. et al. BRAF mutants evade ERK-dependent feedback by different mechanisms that determine their sensitivity to pharmacologic inhibition. Cancer Cell 28, 370-383 (2015).

15. Yao, Z. et al. Tumours with class 3 BRAF mutants are sensitive to the inhibition of activated RAS. Nature 548, 234 (2017)

16. Heidorn, S. J. et al. Kinase-dead BRAF and oncogenic RAS cooperate to drive tumor progression through CRAF. Cell 140, 209-221 (2010).

17. Wan, P. T. C. et al. Mechanism of activation of the RAF-ERK signaling pathway by oncogenic mutations of B-RAF. Cell 116, 855-867 (2004).

18. Shelledy P. L. \& Roman P. B. D. Vemurafenib: first-in-class BRAF-mutated inhibitor for the treatment of unresectable or metastatic melanoma. J. Adv. Pract. Oncol. 6, 361-365 (2015).

19. Sosman, J. A. et al. Survival in BRAF V600-mutant advanced melanoma treated with vemurafenib. N. Engl. J. Med. 366, 707-714 (2012).

20. Salami, J. \& Crews, C. M. Waste disposal-an attractive strategy for cancer therapy. Science 355, 1163-1167 (2017).

21. Sakamoto, K. M. et al. Protacs: chimeric molecules that target proteins to the Skp1-Cullin-F box complex for ubiquitination and degradation. Proc. Natl Acad. Sci. USA 98, 8554-8559 (2001)

22. Bondeson, D. P. et al. Catalytic in vivo protein knockdown by small-molecule PROTACs. Nat. Chem. Biol. 11, 611-617 (2015).

23. Bondeson, D. P. et al. Lessons in PROTAC design from selective degradation with a promiscuous warhead. Cell Chem. Biol. 25, 78-87 e75 (2018).

24. Burslem, G. M. et al. Targeting BCR-ABL1 in chronic myeloid leukemia by PROTAC-mediated targeted protein degradation. Cancer Res 79, 4744-4753 (2019).

25. Burslem, G. M. et al. The advantages of targeted protein degradation over inhibition: an RTK case study. Cell Chem. Biol. 25, 67-77 e63 (2018).

26. Lai, A. C. et al. Modular PROTAC design for the degradation of oncogenic BCR-ABL. Angew. Chem. Int. Ed. Engl. 55, 807-810 (2016)

27. Salami, J. et al. Androgen receptor degradation by the proteolysis-targeting chimera ARCC-4 outperforms enzalutamide in cellular models of prostate cancer drug resistance. Commun. Biol. 1, 100 (2018).

28. Sun, B. et al. BET protein proteolysis targeting chimera (PROTAC) exerts potent lethal activity against mantle cell lymphoma cells. Leukemia 32, 343-352 (2018)

29. Hines, J., Lartigue, S., Dong, H., Qian, Y. \& Crews, C. M. MDM2-recruiting PROTAC offers superior, synergistic antiproliferative activity via simultaneous degradation of BRD4 and stabilization of p53. Cancer Res. 79, 251-262 (2019).

30. Lu, J. et al. Hijacking the E3 ubiquitin ligase cereblon to efficiently target BRD4. Chem. Biol. 22, 755-763 (2015).

31. Smith, B. E. et al. Differential PROTAC substrate specificity dictated by orientation of recruited E3 ligase. Nat. Commun. 10, 131 (2019).

32. Buhimschi, A. D. et al. Targeting the C481S ibrutinib-resistance mutation in Bruton's tyrosine kinase using PROTAC-mediated degradation. Biochemistry 57, 3564-3575 (2018).

33. Karoulia, Z., Gavathiotis, E. \& Poulikakos, P. I. New perspectives for targeting RAF kinase in human cancer. Nat. Rev. Cancer 17, 676-691 (2017).

34. Bollag, G. et al. Clinical efficacy of a RAF inhibitor needs broad target blockade in BRAF-mutant melanoma. Nature 467, 596-599 (2010).

35. Van Molle, I. et al. Dissecting fragment-based lead discovery at the von Hippel-Lindau protein:hypoxia inducible factor lalpha protein-protein interface. Chem. Biol. 19, 1300-1312 (2012).

36. Meng, L. et al. Epoxomicin, a potent and selective proteasome inhibitor, exhibits <em $>$ in vivo $</ \mathrm{em}>$ antiinflammatory activity. Proc. Natl Acad. Sci. 96, 10403-10408 (1999). 
37. Soucy, T. A. et al. An inhibitor of NEDD8-activating enzyme as a new approach to treat cancer. Nature 458, 732-736 (2009).

38. Poulikakos, P. I. et al. RAF inhibitor resistance is mediated by dimerization of aberrantly spliced BRAF(V600E). Nature 480, 387-390 (2011).

39. Lin, L. et al. Mapping the molecular determinants of BRAF oncogene dependence in human lung cancer. Proc. Natl Acad. Sci. USA 111, E748-E757 (2014).

40. Yao, Z. et al. RAF inhibitor PLX8394 selectively disrupts BRAF dimers and RAS-independent BRAF-mutant-driven signaling. Nat. Med. 25, 284-291 (2019).

41. Sen, B. et al. Kinase-impaired BRAF mutations in lung cancer confer sensitivity to dasatinib. Sci. Transl. Med. 4, 136ra170 (2012).

42. Lavoie, H. \& Therrien, M. Regulation of RAF protein kinases in ERK signalling. Nat. Rev. Mol. Cell Biol. 16, 281-298 (2015).

43. Cutler, R. E. Jr, Stephens, R. M., Saracino, M. R. \& Morrison, D. K. Autoregulation of the Raf-1 serine/threonine kinase. Proc. Natl Acad. Sci. USA 95, 9214-9219 (1998).

44. Tran, N. H., Wu, X. \& Frost, J. A. B-Raf and Raf-1 are regulated by distinct autoregulatory mechanisms. J. Biol. Chem. 280, 16244-16253 (2005).

45. Posternak, G. et al. Functional characterization of a PROTAC directed against BRAF mutant V600E. Nat. Chem. Biol. 16, 1170-1178 (2020).

46. Han, X.-R. et al. Discovery of Selective Small Molecule Degraders of BRAFV600E. J. Med. Chem. 63, 4069-4080 (2020).

47. Diedrich, B. et al. Discrete cytosolic macromolecular BRAF complexes exhibit distinct activities and composition. EMBO J. 36, 646-663 (2017).

48. Ritt, D. A., Monson, D. M., Specht, S. I. \& Morrison, D. K. Impact of feedback phosphorylation and Raf heterodimerization on normal and mutant B-Raf signaling. Mol. Cell Biol. 30, 806-819 (2010).

49. Lito, P. et al. Relief of profound feedback inhibition of mitogenic signaling by RAF inhibitors attenuates their activity in BRAFV600E melanomas. Cancer Cell 22, 668-682 (2012).

50. Hatzivassiliou, G. et al. Mechanism of MEK inhibition determines efficacy in mutant KRAS- versus BRAF-driven cancers. Nature 501, 232-236 (2013).

51. Lito, P. et al. Disruption of CRAF-mediated MEK activation is required for effective MEK inhibition in KRAS mutant tumors. Cancer Cell 25, 697-710 (2014).

52. Morris, E. J. et al. Discovery of a novel ERK inhibitor with activity in models of acquired resistance to BRAF and MEK inhibitors. Cancer Discov. 3, 742-750 (2013).

53. Miura, G. MEK it work. Nat. Chem. Biol. 9, 601-601 (2013).

54. Röck, R. et al. BRAF inhibitors promote intermediate BRAF(V600E) conformations and binary interactions with activated RAS. Sci. Adv. 5, eaav8463 (2019).

\section{Acknowledgements}

We thank all members of the Crews and Rosen labs for helpful discussions. We are thankful for Jemilat Salami-Oyenuga, Ashton Lai, George Burslem, Anna Wadhwa, Hanqing Dong, Kanak Raina, and Alicia Morgan for early screening of BRAF-targeting
PROTACs, technical expertise, and meaningful discussion. C.M.C. gratefully acknowledges support from the NIH (R35CA197589) and the American Cancer Society. S.A. is supported by the HHMI Gilliam Fellowship. J.H. is supported by an R50 Research Specialists award from the NCI (R50 CA211252-02).

\section{Author contributions}

C.M.C. and N.R. conceived the project and supervised experiments. S.J.F. designed and synthesized the PROTACS. S.A., Y.G., Z.Y., J.H., K.T.G.S. and L.V. planned and carried out the biological experiments. S.A. wrote the manuscript with assistance from all authors.

\section{Competing interests}

C.M.C. is a consultant and shareholder in Arvinas, Inc, which provides support to his laboratory. N.R. is on the SAB and owns equity in Beigene, Zai Labs, MAPCure, Ribon and Fortress. N.R. is on the SAB of Astra-Zeneca, Chugai, consults with Novartis, Boehringer Ingelheim, RevMed, Eli Lilly and Array-Pfizer and owns equity in Kura. N.R. receives research support from Boerhinger-Ingelheim, Astra-Zeneca, and RevMed. Other authors do not have competing interests.

\section{Additional information}

Supplementary information The online version contains supplementary material available at https://doi.org/10.1038/s41467-021-21159-7.

Correspondence and requests for materials should be addressed to C.M.C.

Peer review information Nature Communications thanks the anonymous reviewers for their contribution to the peer review of this work.

Reprints and permission information is available at http://www.nature.com/reprints

Publisher's note Springer Nature remains neutral with regard to jurisdictional claims in published maps and institutional affiliations.

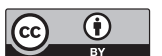

Open Access This article is licensed under a Creative Commons Attribution 4.0 International License, which permits use, sharing, adaptation, distribution and reproduction in any medium or format, as long as you give appropriate credit to the original author(s) and the source, provide a link to the Creative Commons license, and indicate if changes were made. The images or other third party material in this article are included in the article's Creative Commons license, unles indicated otherwise in a credit line to the material. If material is not included in the article's Creative Commons license and your intended use is not permitted by statutory regulation or exceeds the permitted use, you will need to obtain permission directly from the copyright holder. To view a copy of this license, visit http://creativecommons.org/ licenses/by/4.0/.

(c) The Author(s) 2021 